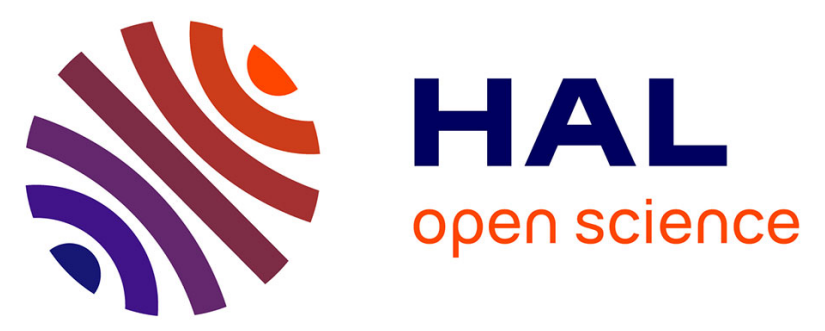

\title{
Structural analysis of the partial state and input observability for structured linear systems. Application to distributed Systems
}

Taha Boukhobza, Frédéric Hamelin, Sinuhé Martinez-Martinez, Dominique Sauter

\section{To cite this version:}

Taha Boukhobza, Frédéric Hamelin, Sinuhé Martinez-Martinez, Dominique Sauter. Structural analysis of the partial state and input observability for structured linear systems. Application to distributed Systems. European Journal of Control, 2009, 15 (5), pp.503-517. hal-00422105

\section{HAL Id: hal-00422105 \\ https://hal.science/hal-00422105}

Submitted on 5 Oct 2009

HAL is a multi-disciplinary open access archive for the deposit and dissemination of scientific research documents, whether they are published or not. The documents may come from teaching and research institutions in France or abroad, or from public or private research centers.
L'archive ouverte pluridisciplinaire HAL, est destinée au dépôt et à la diffusion de documents scientifiques de niveau recherche, publiés ou non, émanant des établissements d'enseignement et de recherche français ou étrangers, des laboratoires publics ou privés. 


\title{
Structural analysis of the partial state and input observability for structured linear systems. Application to distributed Systems
}

\author{
T. Boukhobza, F. Hamelin, S. Martinez-Martinez and D. Sauter \\ Centre de Recherche en Automatique de Nancy (CRAN), Nancy-University, CNRS UMR 7039 \\ BP 70239, 54506 Vandouvre Cedex, Nancy, France, \\ email: taha.boukhobza@cran.uhp-nancy.fr
}

\begin{abstract}
This paper deals with the partial state and input observability analysis for structured linear systems with an application to distributed systems. The proposed method is based on a graph-theoretic approach and assumes only the knowledge of the system's structure. More precisely, we express, in simple graphic terms, necessary and sufficient conditions for the strong observability of a state or an input component. These results are then directly applied to study the observability of a distributed system in some different configurations. In fact, we define two configurations called decentralized interconnected observation scheme and decentralized autonomous observation scheme, for which we check whether or not any given part of the states or the inputs of a considered subsystem is strongly observable. All the provided conditions are easy to verify because they are based on comparison of integers and on finding paths in a digraph.
\end{abstract}

Key words: State and input observability, structured linear systems, distributed systems, graph theory.

\section{Introduction}

The problem of reconstructing any desired part of the states and/or the unknown inputs is also of a great interest in control law synthesis, fault detection and isolation, fault tolerant control, supervision .... Indeed, in many applications, the estimation of only a part of the states and the unknown inputs of the system is necessary to design for example a control law (unmeasured state feedback), a fault detection scheme or a reduced observer. This is also the case, for example, when we want to study the state reconstructubility in the context of a system submitted to faults or non negligible disturbances or in the case where we have to estimate the fault intensity or amplitude in order to appreciate the 
gravity of a malfunction on the system, to design a fault tolerant controller, reject some disturbances or in order to reconfigure the controller in function of the intensity of faults occurring on the system or to know whether or not the state is (or remains) observable when the system is faulty.

In this respect, many works $[5,6,15,19,28,29]$ are focused on the design of full or reduced state observers for linear systems with unknown inputs. Otherwise, the issue of simultaneously observing the whole state and the unknown input has been investigated in $[10,18]$. In this context, a preliminary step to the observer design is the analysis of the state and input observability. Among the most important works dealing with the state and/or input observability, we can cite $[1,2,9,14,27]$ which deal with algebraic or geometric frameworks. In particular, in [9] the author gives the definitions of strong detectability and strong observability and the conditions for existence of observers that estimate a functional of the state and unknown inputs.

Otherwise, many studies deal with the observation of decentralized systems. In this way, in the early 70's, [26] propose, under some decoupling assumptions, the design of a filter for interconnected dynamical systems in which the information pattern is decentralized. More recently, in [25], under some "matching conditions", the authors propose a method for the design of a decentralized reduced state estimator using unknown input observers for large scale systems composed by interconnected systems. Also on the basis of unknown input observers. In [11], a decentralized state function observer is designed for large scale interconnected systems. Three kinds of interconnections are considered and the design of the state function local observer is done under the solvability of some matrix algebraic equations.

In the latter papers as in most other, the studies on the state or/and input observability or on decentralized systems deal with algebraic and geometric tools. The use of such tools requires the exact knowledge of the state space matrices characterizing the system's model. However, in many modeling problems, only zero entries of these matrices, which are determined by means of physical laws, are fixed while the remaining entries are not precisely known. To study the properties of these systems in spite of poor knowledge we have on them, the idea is that we only keep the zero/non-zero entries in the state space matrices. Thus, we consider models where the fixed zeros are conserved while the non-zero entries are replaced by free parameters. There is a huge amount of interesting works in the literature using this kind of models called structured models. The analysis of such systems requires a low computational burden which allows us to deal with large scale systems. Many studies on structured systems are related to the graph-theoretic approach to analyse some system properties such as controllability, observability or the solvability of several classical control problems including distur- 
bance rejection, input-output decoupling, ... [8]. It results from these works that the graph-theoretic approach provides simple and elegant solutions.

However, the well-known graphic observability conditions for linear structured systems recalled in $[8,23,24]$ cannot be applied to systems with unknown inputs. Otherwise, in [3], graphic necessary and sufficient conditions for the input and state observability are given. Nevertheless, these conditions concerns only the whole state and input observability and cannot be applied to know either or not a particular state or an input component is strongly observable. Consequently, for the partial state and input observability, these results are not applicable. Besides, authors of [4] express, in graphic terms, necessary and sufficient conditions for the observability of any given part of a descriptor system states. These results are obviously applicable to the partial state and input observability analysis of linear systems. Nevertheless, the proposed conditions are quite complicated and not efficient from a computational point of view.

In this context, the purpose of this paper is to use a graph-theoretic approach for providing necessary and sufficient conditions which ensure that some particular input and/or state components are generically strongly observable. More exactly, we characterize, for a structured linear system, the set of all the strongly observable state and input components. Next, we use this result to provide necessary and sufficient conditions for the generic observability of structured distributed systems according to their configuration.

Distributed systems are in general composed of a large number of interconnected devices or subsystems. In the last decades, these subsystems can exchange data through communication networks that can be dedicated to the application or not. Examples include industrial automation, building supervision, automotive control, .... Distributed systems provide many advantages such as modular and flexible system and controller designs. However, from an observation point of view, the fact that all the measurements done on the system are not transmitted to all the subsystems can be annihilate the global observability property. In fact, the observability of the state and the reconstructibility of the input components of each subsystem are ones of the main properties which are strongly linked to the data exchanged by the subsystems constituting the considered distributed system and its network configuration. In fact, the observability of each subsystem is linked to the system's subdivision, which redefine the unknown inputs for each subsystem, and to the measurements arriving through the network to each subsystem. Thus, starting from an observable global system is observable, we can obtain, when we subdivide it, non observable subsystems because of unknown inputs which can appear on these subsystems consecutively to the subdivision. This can be true even if all the inputs of the system 
are measured when these measures are not all transmitted through the network to all the subsystems. Hence, an observability analysis of distributed systems, for different network configurations and as function of the information exchanged in the network, is important for the observer design and more generally for the system design. This analysis, for each subsystem, amounts to study the observability of a given part of the states and, necessarily in the most general case, the unknown inputs of linear systems. This is the objective of the second part of the paper.

Note finally that our method is mainly focused on the analysis stage and we do not deal with the observer design problem.

The paper is organised as follows: after Section 2, which is devoted to the problem formulation, a digraph representation of structured systems is given in Section 3. The main results are presented in Section 4 which is mainly subdivided into two parts. At first, the partial state and input observability conditions are given. Next, we treat the analysis of the strong observability in the context of structured distributed systems. Finally, some concluding remarks end the paper.

\section{Problem statement}

In this paper, we treat numerically non-specified systems on the form:

$$
\Sigma_{\Lambda}:\left\{\begin{array}{l}
\dot{x}(t)=A x(t)+B u(t) \\
y(t)=C x(t)+D u(t)
\end{array}\right.
$$

where $x(t) \in \mathbb{R}^{n}, u(t) \in \mathbb{R}^{q}$ and $y(t) \in \mathbb{R}^{p}$ are respectively the state vector, the unknown input vector and the output vector. $A, B, C$ and $D$ represent matrices which elements are either fixed to zero or assumed to be free nonzero parameters noted $\lambda_{i}$. These parameters form a vector $\Lambda=\left(\lambda_{1}, \ldots, \lambda_{h}\right)^{T} \in \mathbb{R}^{h}$. If all parameters $\lambda_{i}$ are numerically fixed, we obtain a so-called admissible realization of structured system $\Sigma_{\Lambda}$. We say that a property is true generically if it is true for almost all the realizations of structured system $\Sigma_{\Lambda}$. Here, "for almost all the realizations" is to be understood as " for all parameter values $\left(\Lambda \in \mathbb{R}^{h}\right)$ except for those in some proper algebraic variety in the parameter space". The proper algebraic variety for which the property is not true is the zero set of some nontrivial polynomial with real coefficients in the $h$ system parameters $\lambda_{1}, \lambda_{2}, \ldots, \lambda_{h}$.

In a first part of this paper, we study the generic partial state and input strong observability for structured system $\Sigma_{\Lambda}$. The notion of and state input strong observability is related to both the strong observability and the left invertibility properties [27] and it is similar to the right-hand side observability 
in the context of descriptor systems [12]. It guarantees that the considered inputs and states part can be expressed only as function of the outputs and their derivatives and so that it is reconstructible using a generalized observer [13].

More precisely, we are interested in the generic strong observability of any given part of the states or the inputs which can be defined as follows:

Definition 1 Consider structured system $\Sigma_{\Lambda}$, we say that a state component $x_{i}(t)$ (respectively an input component $\left.u_{j}(t)\right)$ is generically strongly observable if $y(t)=0, \forall t \geq 0$ implies $x_{i}(t)=0$, $\forall t \geq 0$ (respectively $u_{j}(t)=0, \forall t>0$ ).

Roughly speaking, the generic strong observability of a state component $x_{i}(t)$ (respectively an input component $u_{j}(t)$ ) means that a change in $x_{i}(0)$ (respectively $u_{j}\left(0^{+}\right)$) is necessarily reflected in a change of the measurements.

The first objective of the paper is to give graphic conditions to analyse, for structured system $\Sigma_{\Lambda}$, the question whether or not state component $x_{i}(t)$ or input component $u_{j}(t)$ is generically strongly observable.

In a second stage, we assume that the structured linear system $\Sigma_{\Lambda}$ is a decentralized interconnected system. It is then constituted of several subsystems $\Sigma_{R}^{i}, i=1, \ldots, N$. This subdivision of $(\Sigma)$ into subsystems $\left(\Sigma_{i}\right), i=1, \ldots, N$ will not be discussed in this note but it may be based on geographical repartition of the equipments for example.

The fact is that the global system is distributed and so decomposed into subsystems, each of which being in charge of a local control/observation/supervision/FDI/FTC unit. Some of the information collected by each unit is transmitted via the network. This problem concerns for example complex systems, controlled through a network of controllers. Each controller node is dedicated to a subset of components, and communicates with the others. Since the components are physically interconnected, the local control/observation/supervision system must take the transmitted informations into account. In the paper framework, without loss of generality, we consider distributed system so that each subsystem consists in a part of the plant and its dedicated control node. It is then represented by a state vector which is a part of the whole state vector of the global system. Moreover, an input vector is associated with the subsystem. These inputs act implicitly (directly or not) on the subsystem. Otherwise, some measurements are taken locally on each subsystem. Obviously, there exist physical interactions on different subsystems. These interactions are equivalent to say that some of the state or input components related to subsystem $j$ may intervene on the dynamics of the state components of subsystem 
$i$. On the other hand, the measurements taken on the subsystem $i$ can be explicitly sensitive to the state or input components related to other subsystems. In this case it contains a linear combination of state and input components of subsystem $j$. Thus, in general, and without loss of generality, each subsystem satisfies a model of the form:

$$
\Sigma_{R}^{i}:\left\{\begin{array}{l}
\dot{x}^{i}(t)=A_{i} x^{i}(t)+B_{i} u^{i}(t)+\sum_{j=1, j \neq i}^{N} A_{i, j} x^{j}(t)+\sum_{j=1, j \neq i}^{N} B_{i, j} u^{j}(t) \\
y^{i}(t)=C_{i} x^{i}(t)+D_{i} u^{i}(t)+\sum_{j=1, j \neq i}^{N} C_{i, j} x^{j}(t)+\sum_{j=1, j \neq i}^{N} D_{i, j} u^{j}(t)
\end{array}\right.
$$

where for $i=1, \ldots, N, x^{i}(t) \in \mathbb{R}^{n_{i}}, y^{i}(t) \in \mathbb{R}^{p_{i}}$ and $u^{i}(t) \in \mathbb{R}^{q_{i}}$ are respectively the state, the measurements and the input vectors of subsystem $\Sigma_{R}^{i}$. For $i, j=1, \ldots, N, j \neq i$, matrices $A_{i}, B_{i}, A_{i, j}$, $B_{i, j}, C_{i}, D_{i}, C_{i, j}$ and $D_{i, j}$ represent matrices of appropriate dimensions whose elements are either fixed to zero or assumed to be free nonzero parameters. The whole state vector is denoted by $x(t)=$ $\left(\left(x^{1}(t)\right)^{T}, \ldots,\left(x^{N}(t)\right)^{T}\right)^{T}$, the whole input vector is denoted by $u(t)=\left(\left(u^{1}(t)\right)^{T}, \ldots,\left(u^{N}(t)\right)^{T}\right)^{T}$ and the whole output vector is denoted by $y=\left(\left(y^{1}(t)\right)^{T}, \ldots,\left(y^{N}(t)\right)^{T}\right)^{T}$. Even if a state component of a subsystem acts on the dynamics of another subsystem, but we do not consider this state component "as an unknown input" since its dynamics has a known expression. The term "inputs" is only reserved to the variables $u_{k}^{i}(t)$ with unknown dynamics (there is no expression of $\dot{u}_{k}^{i}(t)$ in the global system's model).

Obviously, as we interest to an observation problem, by the term "output" we mean here measurements, which cannot be considered as inputs of other subsystems, so we do not address a problem of "cascade systems". Otherwise, without loss of generality and for the sake of homogeneity, all the inputs are assumed to be unknown and for control input components which are known or equivalently, from a theoretical point of view, measured. This means that we associate a virtual output equation of the form $y_{k^{\prime}}^{i}=u_{k}^{i}$ to these known inputs. Since, we address an observability analysis problem and not a controller design one, this is not a violation the normal practice of sensor location in a controlled system, because we can restrict all our reasoning to the notion of measured and unmeasured variables. In the framework of this paper, the subsystems exchange some informations through a network as it is the case for the networked control systems. Thus, some measurements can arrive to subsystem $\Sigma_{R}^{i}$ from the other subsystems. These measurements are represented by vector denoted $\tilde{y}^{i}(t)$ which is necessarily a sub-vector of the measurement vector $\left(\left(y^{1}(t)\right)^{T}, \ldots,\left(y^{i-1}(t)\right)^{T},\left(y^{i+1}(t)\right)^{T}, \ldots,\left(y^{N}(t)\right)^{T}\right)^{T}$.

We are interested in the generic strong observability of only a given part of the states and the in- 
puts of each subsystem $\Sigma_{R}^{i}, i=1, \ldots, N$, and we consider two cases. In the first one, we assume that subsystem $\Sigma_{R}^{i}$ is linked to the network and can use the measurement vectors $y^{i}(t)$ and $\tilde{y}^{i}(t)$ to reconstruct a given part of its state and input components. We call this case the decentralized interconnected observation scheme. In the second case, we assume that there are no external measurements arriving through the network to $\Sigma_{R}^{i}$. So, subsystem $\Sigma_{R}^{i}$ can only use its own measurement vector $y^{i}(t)$ to reconstruct a given part of its states and inputs. We call this case the decentralized autonomous observation scheme. The latter case can represent a network cut for example. We define now the strong observability of a state or an input component, relatively to these considered observation schemes, as follows:

Definition 2 Consider linear structured system $\Sigma_{\Lambda}$ constituted of several subsystems $\Sigma_{R}^{i}, i=$ $1, \ldots, N$. For $i \in\{1, \ldots, N\}$, we say that state component $x_{k}^{i}(t), k \in\left\{1, \ldots, n_{i}\right\}$ (respectively input component $\left.u_{j}^{i}(t), j \in\left\{1, \ldots, q_{i}\right\}\right)$ is generically strongly observable in a decentralized interconnected observation scheme if having $y^{i}(t)=0$ and $\tilde{y}^{i}(t)=0$ for $t \geq 0$ implies that $x_{k}^{i}(t)=0$, $\forall t \geq 0$ (respectively $u_{j}^{i}(t)=0, \forall t>0$ ).

Similarly, we say that state component $x_{k}^{i}(t)$ (respectively input component $u_{j}^{i}(t)$ ) is generically strongly observable in a decentralized autonomous observation scheme if having $y^{i}(t)=0$ for $t \geq 0$ implies $x_{k}^{i}(t)=0, \forall t \geq 0$ (respectively $\left.u_{j}^{i}(t)=0, \forall t>0\right)$.

Roughly speaking, a state or an input component of subsystem $\Sigma_{R}^{i}$ is observable in a decentralized interconnected (respectively autonomous) observation scheme when it can be expressed only as function of $y^{i}(t)$ and $\tilde{y}^{i}(t)$ (respectively only as function of $y^{i}(t)$ ) and their derivatives.

The main difference between the so-called decentralized and the autonomous observation schemes is that in the latter case, the considered subsystem can only use its own measurement vector $y^{i}(t)$ and its derivatives to reconstruct some desired unknown variables. On the other hand, in the interconnected observation scheme, the subsystem can use its own measurement $y^{i}(t)$ but also the ones arriving through the network denoted $\tilde{y}^{i}(t)$. The latter may regroup only a part and not the totality of the measurements available on the whole system. That is why, in our study, for the sake of generality, we do not assume that all the subsystems share the same measurement vector $\tilde{y}^{i}(t)$. Note that studying the observability in any of the two considered decentralized schemes can be reduced to two particular cases of a partial state and input observability study.

The two considered observation schemes are called decentralized because they correspond to the case where each subsystem has its own local observer. We do not deal in this paper with a centralized observation case which is equivalent to study the whole input and state observability of a structured 
linear system as it is done using a graph theoretic approach in [3]. The structural observability of a distributed system is then a property which is related to the structure of the global system, to its subdivision and to the information (measurements) available for each subsystem. Hence, this notion which express a sensitivity of the measurements w.r.t. to a change in some initial state or input component is based on the assumption that the global dynamics of the system is known and available for all the subsystems. In the following example, we show first that a global system can be observable while one of its subsystems is not. Next, we illustrate the importance of the global model knowledge for the observability study in distributed schemes.

Example 1 Consider the simple distributed system constituted by the two following subsystems:

$$
\Sigma_{R}^{1}:\left\{\begin{array}{l}
\dot{x}^{1}(t)=\left(\begin{array}{cc}
0 & \lambda_{1} \\
0 & 0
\end{array}\right) x^{1}(t)+\left(\begin{array}{cc}
0 & \lambda_{2} \\
0 & 0
\end{array}\right) x^{2}(t) \\
y^{1}(t)=\left(\begin{array}{cc}
\lambda_{3} & 0
\end{array}\right) x^{1}(t)
\end{array}, \quad \Sigma_{R}^{2}:\left\{\begin{array}{l}
\dot{x}^{2}(t)=\left(\begin{array}{cc}
\lambda_{4} & 0 \\
\lambda_{5} & \lambda_{6}
\end{array}\right) x^{2}(t) \\
y^{2}(t)=\left(\begin{array}{ll}
\lambda_{7} & 0
\end{array}\right) x^{2}(t)
\end{array}\right.\right.
$$

First, note that the global system is obviously generically observable in a centralized observation scheme. Nevertheless, subsystem $\Sigma_{R}^{2}$ is not generically observable in autonomous observation scheme. Indeed, it is not possible to express $x_{2}^{2}(t)$ only as function of $y_{1}^{2}(t)(t)$ and its derivatives. Concerning subsystem $\Sigma_{R}^{1}$, $x_{1}^{1}$ is obviously observable. The objective now is to know if $x_{2}^{1}(t)$ is generically strongly observable. Simple calculations allow to write $x_{2}^{1}(t)=\frac{\dot{y}^{1}(t)}{\lambda_{3} \lambda_{1}}-\frac{\left(\lambda_{4}+\lambda_{5}\right) \ddot{y}^{1}(t)-y^{1(3)}}{\lambda_{4} \lambda_{6} \lambda_{1}}$. We can then conclude that $x_{2}^{1}(t)$ is generically strongly observable. To establish the relation above, we obviously use equations $\dot{x}_{1}^{2}(t)=\lambda_{4} x_{1}^{2}(t)$ and $\dot{x}_{2}^{2}(t)=\lambda_{5} x_{1}^{2}(t)+\lambda_{6} x_{2}^{2}(t)$ termed from model $\Sigma_{R}^{2}$ but not the output equation $y^{2}(t)=\lambda_{7} x_{1}^{2}(t)$. Indeed, it is not possible to express $x_{2}^{1}(t)$ as function of $y^{1}(t)$ and its derivatives without using the dynamics of $x^{2}(t)$.

This example shows two important things. The first is that a system may be observable while one or more of its subsystems are not observable. Next, we illustrate the importance to use the knowledge of the global model (only dynamics part) to establish the observability of some state or input components of a subsystem. Thus, the dimension of an observer which estimate the state of a subsystem may be greater than the dimension of this subsystem. Finally, it is important to note that using the knowledge of the global system's dynamics to establish the observability is different from considering a centralized observation scheme. Indeed, in this example, we use the $x^{2}(t)$ dynamics to establish the observability of $x_{1}^{1}(t)$ but we do not use the output equation $y^{2}(t)$. 
As it is shown in the above example, checking the observability as it is specified in Definition 2 implies the possible use of the global dynamical equations of the system. Indeed, in such definition, the unknown input of the $i^{\text {th }}$ subsystem is the whole input vector $u(t)$ and not the state components of the other subsystems $x^{j}(t), j \neq i$. To make this property meaningful in a practical sense, it is then necessary to assume that, for each subsystem, the reconstruction of the state and the unknown input components may employ any useful dynamical equation given in the global model of the system and not only a reduced model defined by the equations of the considered subsystem. Due to the actual high performances of the calculators used to implement practically different observers and estimators, we can assume, that the global dynamics of the whole system's state components is available for all the subsystems and can be used as a common knowledge. In this context, in both the studied cases, we assume that we can implement locally for each subsystem an "extended" observer i.e. an observer which is designed on the basis of the needed part of the whole system dynamics. It implies that the state dimension of a local observer may be bigger than the state dimension of the considered subsystem. Obviously, the main drawback of assuming in Definition 2, the unknown input for all the subsystems represented by $u(t)$, is that it makes, perhaps, quite necessary the implementation a large part of the global model of the system in each local observer. Note that, depending on the measured variables and on the transmitted measures, only a part of the global system's dynamics may be sufficient to the local observers to reconstruct the desired states and components.

When we deal with a large scale or a complex system, this drawback can become severe. This is why, at the end of the paper, we consider the case where for the $i^{\text {th }}$ subsystem, the state components of all the other subsystems are considered as unknown inputs. We obtain two new observation schemes called reduced interconnected and reduced autonomous observation schemes.

Note finally that analysing the observability property in any of all these observation schemes (centralized, interconnected, reduced) can be done by applying only one theoretical result that is the graphical characterization of the partial strong state and input observability.

Therefore, the aim of the paper is to give graphical conditions to answer the question whether or not a given state or input component is strongly observable for a linear structured system $\Sigma_{\Lambda}$ and next for any of the considered observation schemes in the case of distributed systems. In this respect, the next section is dedicated to the definition of a graphical representation of linear structured systems and to some notations. 


\section{Graphical representation of structured linear systems}

For the sake of simplicity, we consider at first the case of a simple linear structured system $\Sigma_{\Lambda}$. The following definitions are adapted to the case of distributed systems in subsection 4.3.

The directed graph or digraph $\mathcal{G}\left(\Sigma_{\Lambda}\right)(1)$ associated with $\Sigma_{\Lambda}$ is constituted by a vertex set $\mathcal{V}$ and an edge set $\mathcal{E}$ i.e. $\mathcal{G}\left(\Sigma_{\Lambda}\right)=(\mathcal{V}, \mathcal{E})$. The vertices are associated with the state, the unknown input and the output components of $\Sigma_{\Lambda}$ whereas the edges represent links between these variables. More precisely, $\mathcal{V}=\mathbf{X} \cup \mathbf{Y} \cup \mathbf{U}$, where $\mathbf{X}=\left\{\mathbf{x}_{\mathbf{1}}, \ldots, \mathbf{x}_{\mathbf{n}}\right\}$ is the set of state vertices, $\mathbf{Y}=\left\{\mathbf{y}_{\mathbf{1}}, \ldots, \mathbf{y}_{\mathbf{p}}\right\}$ is the set of output vertices and $\mathbf{U}=\left\{\mathbf{u}_{1}, \ldots, \mathbf{u}_{\mathbf{q}}\right\}$ is the set of unknown input vertices. The edge set is $\mathcal{E}=A$-edges $\cup B$-edges $\cup C$-edges $\cup D$-edges, with $A$-edges $=\left\{\left(\mathbf{x}_{\mathbf{j}}, \mathbf{x}_{\mathbf{i}}\right) \mid A(i, j) \neq 0\right\}, B$-edges $=$ $\left\{\left(\mathbf{u}_{\mathbf{j}}, \mathbf{x}_{\mathbf{i}}\right) \mid B(i, j) \neq 0\right\}, C$-edges $=\left\{\left(\mathbf{x}_{\mathbf{j}}, \mathbf{y}_{\mathbf{i}}\right) \mid C(i, j) \neq 0\right\}, D$-edges $=\left\{\left(\mathbf{u}_{\mathbf{j}}, \mathbf{y}_{\mathbf{i}}\right) \mid D(i, j) \neq 0\right\}$, where $M(i, j)$ is the $(i, j)$ th element of matrix $M$ and $\left(\mathbf{v}_{\mathbf{1}}, \mathbf{v}_{\mathbf{2}}\right)$ denotes a directed edge from vertex $\mathbf{v}_{\mathbf{1}} \in \mathcal{V}$ to vertex $\mathbf{v}_{\mathbf{2}} \in \mathcal{V}$.

Hereafter, we illustrate the proposed digraph representation with an example.

Example 2 The system defined by the following matrices is represented by the digraph in Figure 1.

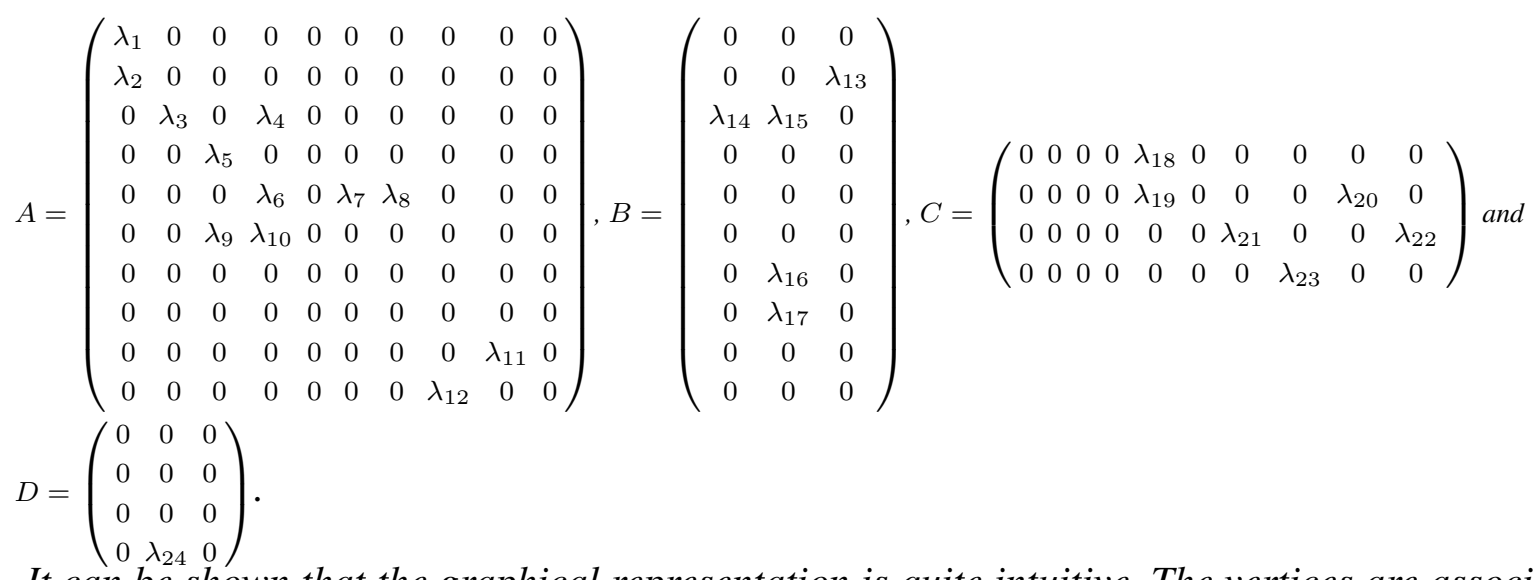

It can be shown that the graphical representation is quite intuitive. The vertices are associated with

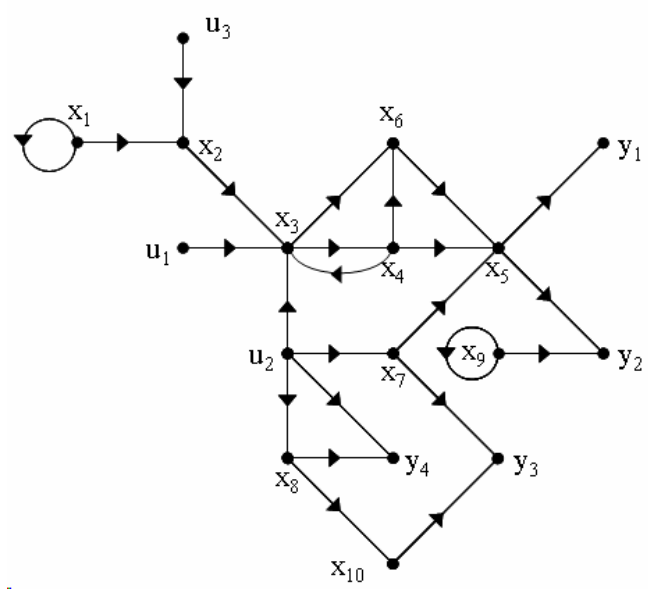

Figure 1. Digraph associated with system of Example 2 
the state, the unknown input and the output components o whereas the edges represent links between these variables. Indeed, for example, $\dot{x}_{3}=\lambda_{3} x_{2}+\lambda_{4} x_{4}+\lambda_{14} u_{1}+\lambda_{15} u_{2}$ is graphically represented by edges starting from vertices $\mathbf{x}_{2}, \mathbf{x}_{4}, \mathbf{u}_{1}$ and $\mathbf{u}_{2}$ and arriving to vertex $\mathbf{x}_{3}$.

Let us now give some useful definitions and notations.

- Two edges $e_{1}=\left(\mathbf{v}_{\mathbf{1}}, \mathbf{v}_{\mathbf{1}}^{\prime}\right)$ and $e_{2}=\left(\mathbf{v}_{\mathbf{2}}, \mathbf{v}_{\mathbf{2}}^{\prime}\right)$ are $v$-disjoint if $\mathbf{v}_{\mathbf{1}} \neq \mathbf{v}_{\mathbf{2}}$ and $\mathbf{v}_{\mathbf{1}}^{\prime} \neq \mathbf{v}_{\mathbf{2}}^{\prime}$. Note that $e_{1}$ and $e_{2}$ can be $v$-disjoint even if $\mathbf{v}_{\mathbf{1}}^{\prime}=\mathbf{v}_{\mathbf{2}}$ or $\mathbf{v}_{\mathbf{1}}=\mathbf{v}_{\mathbf{2}}^{\prime}$.

Some edges are $v$-disjoint if they are mutually $v$-disjoint.

- We denote path $P$ containing vertices $\mathbf{v}_{\mathbf{r}_{0}}, \ldots, \mathbf{v}_{\mathbf{r}_{\mathbf{i}}}$ by $P=\mathbf{v}_{\mathbf{r}_{0}} \rightarrow \mathbf{v}_{\mathbf{r}_{1}} \rightarrow \ldots \rightarrow \mathbf{v}_{\mathbf{r}_{\mathbf{i}}}$, where $\left(\mathbf{v}_{\mathbf{r}_{\mathbf{j}}}, \mathbf{v}_{\mathbf{r}_{\mathbf{j}+\mathbf{1}}}\right) \in \mathcal{E}$ for $j=0,1, \ldots, i-1$. We say that $P$ covers $\mathbf{v}_{\mathbf{r}_{\mathbf{0}}}, \mathbf{v}_{\mathbf{r}_{\mathbf{1}}}, \ldots, \mathbf{v}_{\mathbf{r}_{\mathbf{i}}}$. A path is simple when every vertex occurs only once in this path.

- A cycle is a path of the form $\mathbf{v}_{\mathbf{r}_{0}} \rightarrow \mathbf{v}_{\mathbf{r}_{1}} \rightarrow \ldots \rightarrow \mathbf{v}_{\mathbf{r}_{\mathbf{i}}} \rightarrow \mathbf{v}_{\mathbf{r}_{0}}$, where all vertices $\mathbf{v}_{\mathbf{r}_{0}}, \mathbf{v}_{\mathbf{r}_{1}}, \ldots, \mathbf{v}_{\mathbf{r}_{\mathbf{i}}}$ are distinct.

- Some paths (resp. cycles) are disjoint if they have no common vertex. A set of disjoint cycles is called a cycle family.

- Path $P$ is an $\mathbf{Y}$-topped path if its end vertex is an element of $\mathbf{Y}$. An $\mathbf{Y}$-topped path family consists of disjoint simple $\mathbf{Y}$-topped paths.

- The union of a Y-topped path family and a cycle family is disjoint if they have no vertices in common. If such union contains path or a cycle which covers a vertex $\mathbf{v}$ it is said to cover $\mathbf{v}$.

Let $\mathcal{V}_{1}$ and $\mathcal{V}_{2}$ denote two subsets of $\mathcal{V}$. The cardinality of $\mathcal{V}_{1}$ is noted $\operatorname{card}\left(\mathcal{V}_{1}\right)$. $\mathcal{V}_{1} \backslash \mathcal{V}_{2}$ denotes the set of elements in $\mathcal{V}_{1}$ which are not in $\mathcal{V}_{2}$ i.e. $\mathcal{V}_{1} \backslash \mathcal{V}_{2}=\left\{\mathbf{v} \in \mathcal{V}_{1}\right.$ such that $\left.\mathbf{v} \notin \mathcal{V}_{2}\right\}$

- A path $P=\mathbf{v}_{\mathbf{r}_{0}} \rightarrow \mathbf{v}_{\mathbf{r}_{1}} \rightarrow \ldots \rightarrow \mathbf{v}_{\mathbf{r}_{\mathbf{i}}}$ is said a $\mathcal{V}_{1}-\mathcal{V}_{2}$ path if $\mathbf{v}_{\mathbf{r}_{\mathbf{0}}} \in \mathcal{V}_{1}$ and $\mathbf{v}_{\mathbf{r}_{\mathbf{i}}} \in \mathcal{V}_{2}$. Moreover, if the only vertex of $P$ which belongs to $\mathcal{V}_{1}$ is $\mathbf{v}_{\mathbf{r}_{\mathbf{o}}}$ and the only vertex of $P$ which belongs to $\mathcal{V}_{2}$ is $\mathbf{v}_{\mathbf{r}_{\mathbf{i}}}$, $P$ is called a direct $\mathcal{V}_{1}-\mathcal{V}_{2}$ path.

- A set of $\ell$ disjoint $\mathcal{V}_{1}-\mathcal{V}_{2}$ paths is called a $\mathcal{V}_{1}-\mathcal{V}_{2}$ linking of size $\ell$. The linkings which consist of a maximal number of disjoint $\mathcal{V}_{1}-\mathcal{V}_{2}$ paths are called maximum $\mathcal{V}_{1}-\mathcal{V}_{2}$ linkings. We define by $\rho\left[\mathcal{V}_{1}, \mathcal{V}_{2}\right]$ the size of these maximum $\mathcal{V}_{1}-\mathcal{V}_{2}$ linkings.

- $\mu\left[\mathcal{V}_{1}, \mathcal{V}_{2}\right]$ is the minimal number of vertices covered by a maximum $\mathcal{V}_{1}-\mathcal{V}_{2}$ linking.

- $\theta\left[\mathcal{V}_{1}, \mathcal{V}_{2}\right]$ is the maximal number of $v$-disjoint edges which start in $\mathcal{V}_{1}$ and end in $\mathcal{V}_{2}$.

- The set of all essential vertices for the maximum $\mathcal{V}_{1}-\mathcal{V}_{2}$ linkings, noted $V_{\text {ess }}\left(\mathcal{V}_{1}, \mathcal{V}_{2}\right)$, is defined by $V_{e s s}\left(\mathcal{V}_{1}, \mathcal{V}_{2}\right) \stackrel{\text { def }}{=}\left\{\mathbf{v} \in \mathcal{V} \mid \mathbf{v}\right.$ is included in every maximum $\mathcal{V}_{1}-\mathcal{V}_{2}$ linking $\}$

- Subset $\mathbf{S}\left(\mathcal{V}_{1}, \mathcal{V}_{2}\right) \subseteq \mathcal{V}$ is a separator between sets $\mathcal{V}_{1}$ and $\mathcal{V}_{2}$, if every path from $\mathcal{V}_{1}$ to $\mathcal{V}_{2}$ contains at least one vertex in $\mathbf{S}\left(\mathcal{V}_{1}, \mathcal{V}_{2}\right) \subseteq \mathcal{V}$. We call minimum separators between $\mathcal{V}_{1}$ and $\mathcal{V}_{2}$ any separators 
having the smallest size. There is an uniquely determined minimum separator between $\mathcal{V}_{1}$ and $\mathcal{V}_{2}$ noted $\mathbf{S}^{\mathbf{o}}\left(\mathcal{V}_{1}, \mathcal{V}_{2}\right)$, called minimum output separator [30] and which is the set of start vertices of all direct $V_{\text {ess }}\left(\mathcal{V}_{1}, \mathcal{V}_{2}\right)-\mathcal{V}_{2}$ paths, where $V_{\text {ess }}\left(\mathcal{V}_{1}, \mathcal{V}_{2}\right) \cap \mathcal{V}_{2}$ is considered, in the present definition, as input vertices.

In Example 2, $\rho[\mathbf{U}, \mathbf{Y}]=2$ and $\theta[\mathbf{U}, \mathbf{Y}]=1$. Furthermore, there exist several maximum linkings: $\left\{\mathrm{u}_{1} \rightarrow \mathrm{x}_{3} \rightarrow \mathrm{x}_{4} \rightarrow \mathrm{x}_{5} \rightarrow \mathrm{y}_{1} ; \mathrm{u}_{2} \rightarrow \mathrm{y}_{4}\right\}, \quad\left\{\mathrm{u}_{1} \rightarrow \mathrm{x}_{3} \rightarrow \mathrm{x}_{4} \rightarrow \mathrm{x}_{6} \rightarrow \mathrm{x}_{5} \rightarrow \mathrm{y}_{1} ; \mathrm{u}_{2} \rightarrow \mathrm{x}_{8} \rightarrow \mathrm{y}_{4}\right\}, \ldots$ According to the fact that the first maximum linking is the "shortest one", we have that $\mu[\mathbf{U}, \mathbf{Y}]=7$. Furthermore, $V_{\text {ess }}(\mathbf{U}, \mathbf{Y})=\left\{\mathbf{x}_{\mathbf{3}}, \mathbf{x}_{\mathbf{5}}, \mathbf{u}_{\mathbf{2}}\right\}$ and $\mathbf{S}^{\mathbf{o}}(\mathbf{U}, \mathbf{Y})=\left\{\mathbf{x}_{\mathbf{5}}, \mathbf{u}_{\mathbf{2}}\right\}$.

We give now the following important definition [3]:

Definition 3 For each vertex subset $\mathrm{V}$ such that $\mathrm{Y} \subseteq \mathrm{V} \subseteq \mathbf{X} \cup \mathbf{U} \cup \mathbf{Y}$, we define the following vertex subsets:

- $\mathbf{X}_{\mathbf{1}}(\mathbf{V}) \stackrel{\text { def }}{=}\left\{\mathbf{x}_{\mathbf{i}} \in \mathbf{X} \backslash \mathbf{V} \mid \rho\left[\mathbf{U} \cup\left\{\mathbf{x}_{\mathbf{i}}\right\}, \mathbf{V}\right]>\rho[\mathbf{U}, \mathbf{V}]\right\}$;

- $\mathbf{\Upsilon}_{\mathbf{1}}(\mathbf{V}) \stackrel{\text { def }}{=} \mathbf{V} \backslash V_{\text {ess }}(\mathbf{U}, \mathbf{V})$;

- $\beta_{1}(\mathbf{V})$ is the maximal number of vertices of $\mathbf{X}_{\mathbf{1}}(\mathbf{V}) \cup S^{o}(\mathbf{U}, \mathbf{V}) \backslash \mathbf{V}$ covered by a disjoint union of - $a \mathbf{S} \mathbf{o}(\mathbf{U}, \mathbf{V}) \backslash \mathbf{V}-\Upsilon_{\mathbf{1}}(\mathbf{V})$ linking of maximal size,

- a $\Upsilon_{\mathbf{1}}(\mathrm{V})$-topped path family and

- a cycle family covering only elements of $\mathbf{X}_{\mathbf{1}}(\mathrm{V})$.

- $\beta_{0}(\mathbf{V}) \stackrel{\text { def }}{=} \mu\left[\mathbf{U}, S^{o}(U, \mathbf{V})\right]-\rho\left[\mathbf{U}, S^{o}(U, \mathbf{V})\right]$;

- $\beta(\mathbf{V}) \stackrel{\text { def }}{=} \beta_{1}(\mathbf{V})+\beta_{0}(\mathbf{V})+\operatorname{card}(\mathbf{V} \backslash \mathbf{Y})$.

In fact, the subdivision corresponds to a particular decomposition of the matrix pencil of the system similar to the one suggested in [31] but it is here explicitly defined. Moreover, it is well-adapted to the application of the two main theorems of [30] characterizing the generic rank of the matrix pencil of a structured system in the two particular cases of a square system and a particular rectangular system. To illustrate the previous definition, in the case of the system described in Example 2, we have, $\mathbf{X}_{\mathbf{1}}(\mathbf{Y})=\left\{\mathbf{x}_{\mathbf{7}}, \mathbf{x}_{\mathbf{8}}, \mathbf{x}_{\mathbf{9}}, \mathbf{x}_{\mathbf{1 0}}\right\}, \mathbf{\Upsilon}_{\mathbf{1}}(\mathbf{Y})=\mathbf{Y}, \mathbf{S}^{\mathbf{o}}(\mathbf{U}, \mathbf{Y})=\left\{\mathbf{x}_{\mathbf{5}}\right\}, \beta_{0}(\mathbf{Y})=4-1$. Moreover, the maximal number of state and input vertices covered by the disjoint union of two $\left\{\mathbf{x}_{\mathbf{5}}\right\} \cup \mathbf{U}_{\mathbf{1}}(\mathbf{Y})$ $\Upsilon_{\mathbf{1}}(\mathbf{Y})$ paths and a $\Upsilon_{\mathbf{1}}(\mathbf{Y})$-topped path family which does not cover $\mathbf{x}_{\mathbf{9}}$ is $4: \mathbf{u}_{\mathbf{2}} \rightarrow \mathbf{x}_{\mathbf{8}} \rightarrow \mathbf{x}_{\mathbf{1 0}} \rightarrow \mathbf{y}_{\mathbf{3}}$ and $\mathbf{x}_{\mathbf{5}} \rightarrow \mathbf{y}_{\mathbf{1}}$. So, $\beta_{1}(\mathbf{Y})=5$ and then $\beta(\mathbf{Y})=5+(4-1)+0=8$. 


\section{Main results}

\subsection{Partial strong observability analysis}

Considering structured linear system $\Sigma_{\Lambda}$, this first subsection is devoted to the exact characterization of the set of all the strongly observable input and state components. At this aim, we have recourse to the computation of the generic dimension of the strongly observable subspace in the extended state and input space $\left(x^{T}, u^{T}\right)^{T}$. As we shall see below, this dimension is related to function $\beta$.

In order to simplify the graph by eliminating some unobservable inputs, let us consider, for each vertex subset $\mathbf{V}$ such that $\mathbf{Y} \subseteq \mathbf{V} \subseteq \mathbf{X} \cup \mathbf{U} \cup \mathbf{Y}$, the input vertex subset $\overline{\mathbf{U}}(\mathbf{V}) \subseteq \mathbf{U}$ such that $\operatorname{card}(\overline{\mathbf{U}}(\mathbf{V}))=\rho[\mathbf{U}, \mathbf{V}]=\rho[\overline{\mathbf{U}}(\mathbf{V}), \mathbf{V}]$ and $\mu[\overline{\mathbf{U}}(\mathbf{V}), \mathbf{V}]=\mu[\mathbf{U}, \mathbf{V}]$. Note that $\overline{\mathbf{U}}(\mathbf{V})$ always exists but is not necessarily unique.

Using the results of [7], where authors treat the disturbance rejection problem, we have that input components included in $\mathbf{U} \backslash \overline{\mathbf{U}}(\mathbf{Y})$ can be rendered unobservable using the $\overline{\mathbf{U}}(\mathbf{Y})$ components i.e. there exist inputs associated with the vertices included in $\overline{\mathbf{U}}(\mathbf{Y})$ such that output $y(t)$ is not sensitive to the input components associated with $\mathbf{U} \backslash \overline{\mathbf{U}}(\mathbf{Y})$. Hence, the input components associated with vertices $\mathbf{U} \backslash \overline{\mathbf{U}}(\mathbf{Y})$ are not strongly observable and so, for the sake of simplicity, we restrict our observability study only to the input components associated with $\overline{\mathbf{U}}(\mathbf{Y})$.

Let us denote by $\bar{B}$ (resp. $\bar{D}$ ) the sub-matrix of $B$ (resp. D) associated with $\overline{\mathbf{U}}(\mathbf{Y})$ i.e. matrix $\bar{B}$ (resp. $\bar{D}$ ) is constituted by the concatenation of columns $B_{j}$ (resp. $D_{j}$ ) of $B$ (resp. $D$ ) where $\mathbf{u}_{\mathbf{j}} \in \overline{\mathbf{U}}(\mathbf{Y})$. Let us denote by $q$ the cardinality of $\overline{\mathbf{U}}(\mathbf{Y})$ and the pencil matrix of system $(A, \bar{B}, C, \bar{D})$ by $P(s)=\left(\begin{array}{cc}A-s I_{n} & \bar{B} \\ C & \bar{D}\end{array}\right)$.

Regarding $P(s)$ as a rational matrix, we call its rank the normal-rank [30] and we denote this normal rank by $n$-rank $(P(s))$. Thus, for each realization of $\Sigma_{\Lambda}$, we can compute the $n$-rank of $P(s)$. This rank will have the same value for almost all parameter values $\lambda \in \mathbb{R}^{h}[24,30]$. This so-called generic $n$-rank of $P(s)$ will be denoted by $g \_n$-rank $(P(s))$. The generic rank of matrix $P(s)$, denoted $g \_r a n k(P(s))$, is quite different as it depends on $s$. Hence, $g \_r a n k(P(s))=r, \forall s \in \mathbb{C}$ means that for almost all parameter values $\lambda \in \mathbb{R}^{h}, \operatorname{rank}(P(s))=r, \forall s \in \mathbb{C}$.

The system defined by matrices $(A, \bar{B}, C, \bar{D})$ is generically input and state observable iff $g \_\operatorname{rank}(P(s))=n+\bar{q}$ or in other words iff $P(s)$ has generically full column rank. Otherwise, if all the state and input components are the start vertices of $\mathbf{Y}$-topped paths and as $\rho[\overline{\mathbf{U}}(\mathbf{Y}), \mathbf{Y}]=$ $\operatorname{card}(\overline{\mathbf{U}}(\mathbf{Y}))=\bar{q}$, using the results of [30], we have that the generic normal rank of $P(s)$ is equal to 
$n+\bar{q}$. Thus, $g \_r a n k\left(P\left(s_{0}\right)\right)<n+\bar{q}$ is equivalent [27] to the existence of a nonzero vector $\left(x_{0}^{T}, u_{0}^{T}\right)^{T}$ such that the output $y(t)$ resulting from the input $u(t)=u_{0} e^{s_{0} t}$ and the initial condition $x(0)=x_{0}$ is zero and so that there exists a linear combination of the extended state and input components that is not strongly observable. Consequently, the generic dimension of the strongly observable subspace in the extended state and input space $\left(x^{T}(t), u^{T}(t)\right)^{T}$ is equal to $n+\operatorname{card}(\overline{\mathbf{U}}(\mathbf{Y}))-g_{-} n_{\text {inv, } z}$, where $g_{-} n_{i n v, z}$ denotes the generic number of invariant zeros of $P(s)$, defined as the complex solutions of inequality g_rank $(P(s))<n+\bar{q}$ [27].

Before giving a necessary and sufficient condition for the strong observability of a state or an input component, we give hereafter a graphical characterization of $g \_n_{i n v, z}$.

Obviously, to compute $g_{-} n_{i n v, z}$, we can use the results provided in [31] which complete the ones presented in [30]. However, on the one hand, the subdivision of the studied system into three subsystems suggested in [31] is not necessary to study the observability because of the simplification induced by considering as input vertices $\overline{\mathbf{U}}(\mathbf{Y})$ instead of $\mathbf{U}$.

The following lemma gives a graphical characterization of $g_{-} n_{i n v, z}$ :

Lemma 1 Consider structured system $\Sigma_{\Lambda}$ represented by digraph $\mathcal{G}\left(\Sigma_{\Lambda}\right)$. We have that $n+\bar{q}-$ $g \_n_{i n v, z}=\beta(\mathbf{Y})$, where $g \_n_{i n v, z}$ is the generic number of invariant zeros of $P(s)$.

Proof: Let us first complete the subdivision presented in Definition 3:

- $\Upsilon_{\mathbf{0}}(\mathbf{V}) \stackrel{\text { def }}{=} \mathbf{V} \backslash \Upsilon_{\mathbf{1}}(\mathbf{V})$;

- $\mathbf{U}_{\mathbf{0}}(\mathbf{V}) \stackrel{\text { def }}{=}\left\{\mathbf{u}_{\mathbf{i}} \in \overline{\mathbf{U}}(\mathbf{V}) \mid \theta\left[\left\{\mathbf{u}_{\mathbf{i}}\right\}, \mathbf{X}_{\mathbf{1}}(\mathbf{V}) \cup \boldsymbol{\Upsilon}_{\mathbf{1}}(\mathbf{V})\right]=0\right\}$;

- $\mathbf{U}_{\mathbf{1}}(\mathbf{V}) \stackrel{\text { def }}{=} \overline{\mathbf{U}}(\mathbf{V}) \backslash \mathbf{U}_{\mathbf{0}}(\mathbf{V})$;

- $\mathbf{X}_{\mathbf{s}}(\mathbf{V}) \stackrel{\text { def }}{=}\left(\mathbf{S}^{\mathbf{o}}(\mathbf{U}, \mathbf{V}) \cap \mathbf{X}\right) \backslash \mathbf{V}$;

- $\mathbf{X}_{\mathbf{0}}(\mathbf{V}) \stackrel{\text { def }}{=} \overline{\mathbf{X}} \backslash\left(\mathbf{X}_{\mathbf{1}}(\mathbf{V}) \cup \mathbf{X}_{\mathbf{s}}(\mathbf{V}) \cup \mathbf{V}\right)$;

In [3], the subdivision of the system described above is introduced. Mainly, it some particular properties: $V_{\text {ess }}(\overline{\mathbf{U}}(\mathbf{V}), \mathbf{V})=V_{\text {ess }}\left(\mathbf{U}_{\mathbf{0}}(\mathbf{V}), \mathbf{V}\right) \cup \mathbf{U}_{\mathbf{1}}(\mathbf{V}), \theta\left[\mathbf{X}_{\mathbf{s}}(\mathbf{V}), \mathbf{X}_{\mathbf{1}}(\mathbf{V}) \cup \boldsymbol{\Upsilon}_{\mathbf{1}}(\mathbf{V})\right]=\operatorname{card}\left(\mathbf{X}_{\mathbf{s}}(\mathbf{V})\right)$, $\mathbf{S}^{\mathbf{o}}(\overline{\mathbf{U}}(\mathbf{V}), \mathbf{V})=\mathbf{X}_{\mathbf{s}}(\mathbf{V}) \cup \boldsymbol{\Upsilon}_{\mathbf{0}}(\mathbf{V}) \cup \mathbf{U}_{\mathbf{1}}(\mathbf{V})$ and $\theta\left[\mathbf{X}_{\mathbf{0}}(\mathbf{V}) \cup \boldsymbol{\Upsilon}_{\mathbf{0}}(\mathbf{V}), \mathbf{X}_{\mathbf{1}}(\mathbf{V}) \cup \boldsymbol{\Upsilon}_{\mathbf{1}}(\mathbf{V})\right]=0$.

Consider a vertex set $\mathbf{V}$ such that $\mathbf{Y} \subseteq \mathbf{V} \subseteq \mathbf{X} \cup \mathbf{U} \cup \mathbf{Y}$ and assume that the measurements of the system are extended to the components associated with $\mathbf{V}$. The latter equalities allow us to study the input and state observability of such extended system $\Sigma_{\Lambda}$ by considering independently two systems: - a square system denoted $\Sigma_{0}$, defined by input $\mathbf{U}_{\mathbf{0}}(\mathbf{V})$, state $\mathbf{X}_{\mathbf{0}}(\mathbf{V})$ and output $\mathbf{X}_{\mathbf{s}}(\mathbf{V}) \cup \Upsilon_{\mathbf{0}}(\mathbf{V})$, - a system denoted $\Sigma_{1}$, defined by input $\mathbf{U}_{\mathbf{1}}(\mathbf{V}) \cup \mathbf{X}_{\mathbf{s}}(\mathbf{V})$, state $\mathbf{X}_{\mathbf{1}}(\mathbf{V})$ and output $\Upsilon_{\mathbf{1}}(\mathbf{V})$. 
The particularity of the proposed subdivision is that the input and state observability of $\Sigma_{\Lambda}$ can be done by studying separately $\Sigma_{0}$ and $\Sigma_{1}[3]$.

Mainly, we have that there is no edge from $\mathbf{X}_{\mathbf{0}}(\mathbf{Y}) \cup \mathbf{U}_{\mathbf{0}}(\mathbf{Y})$ to $\mathbf{X}_{\mathbf{1}}(\mathbf{Y}) \cup \Upsilon_{\mathbf{1}}(\mathbf{Y})$ and $\mathbf{S}^{\mathbf{o}}\left(\mathbf{U}_{\mathbf{0}}(\mathbf{Y}), \mathbf{Y}\right)=$ $\mathbf{X}_{\mathbf{s}}(\mathbf{Y}) \cup \Upsilon_{\mathbf{0}}(\mathbf{Y})$. Thus, we can write $\Sigma_{\Lambda}$ as:

$$
\left\{\begin{array}{l}
\dot{x}_{0}(t)=A_{0,0} x_{0}(t)+A_{0, s} x_{s}(t)+A_{0,1} x_{1}(t)+B_{0,0} u_{0}(t)+B_{0,1} u_{1}(t) \\
\dot{x}_{s}(t)=A_{s, 0} x_{0}(t)+A_{s, s} x_{s}(t)+A_{s, 1} x_{1}(t)+B_{s, 0} u_{0}(t)+B_{s, 1} u_{1}(t) \\
\dot{x}_{1}(t)=A_{1, s} x_{s}(t)+A_{1,1} x_{1}(t)+B_{1,1} u_{1}(t) \\
v_{0}(t)=C_{0,0} x_{0}(t)+C_{0, s} x_{s}(t)+C_{0,1} x_{1}(t)+D_{0,0} u_{0}(t)+D_{0,1} u_{1}(t) \\
v_{1}(t)=C_{1, s} x_{s}(t)+C_{1,1} x_{1}(t)+D_{1,1} u_{1}(t)
\end{array}\right.
$$

where $x_{0}(t), x_{s}(t), x_{1}(t), y_{0}(t), y_{1}(t), v_{0}(t)$ and $v_{1}(t)$ represent the variables associated with vertex subsets $\mathbf{X}_{\mathbf{0}}(\mathbf{Y}), \mathbf{X}_{\mathbf{s}}(\mathbf{Y}), \mathbf{X}_{\mathbf{1}}(\mathbf{Y}), \mathbf{U}_{\mathbf{0}}(\mathbf{Y}), \mathbf{U}_{\mathbf{1}}(\mathbf{Y}), \Upsilon_{\mathbf{0}}(\mathbf{Y})$ and $\Upsilon_{\mathbf{1}}(\mathbf{Y})$ respectively.

For the sake of simplicity, let us define $n_{0}=\operatorname{card}\left(\mathbf{X}_{\mathbf{0}}(\mathbf{Y})\right), n_{s}=\operatorname{card}\left(\mathbf{X}_{\mathbf{s}}(\mathbf{Y})\right), n_{1}=\operatorname{card}\left(\mathbf{X}_{\mathbf{1}}(\mathbf{Y})\right)$, $q_{0}=\operatorname{card}\left(\mathbf{U}_{\mathbf{0}}(\mathbf{Y})\right), q_{1}=\operatorname{card}\left(\mathbf{U}_{\mathbf{1}}(\mathbf{Y})\right), p_{0}=\operatorname{card}\left(\mathbf{\Upsilon}_{\mathbf{0}}(\mathbf{Y})\right)$ and $p_{1}=\operatorname{card}\left(\mathbf{\Upsilon}_{\mathbf{1}}(\mathbf{Y})\right)$.

Starting from system (3), with some appropriate permutations on the rows and columns of $P(s)$, we can transform $P(s)$ into

$$
\tilde{P}(s)=\left(\begin{array}{ccccc}
A_{0,0}-s I_{n_{0}} & A_{0, s} & B_{0,0} & A_{0,1} & B_{0,1} \\
A_{s, 0} & A_{s, s}-s I_{n_{s}} & B_{s, 0} & A_{s, 1} & B_{s, 1} \\
C_{0,0} & C_{0, s} & D_{0,0} & C_{0,1} & D_{0,1} \\
0 & A_{1, s} & 0 & A_{1,1}-s I_{n_{1}} & B_{1,1} \\
0 & C_{1, s} & 0 & C_{1,1} & D_{1,1}
\end{array}\right)
$$

Since the edges associated with $A_{1, s} \operatorname{link} \mathbf{X}_{\mathbf{s}}(\mathbf{Y})$ to $\mathbf{X}_{\mathbf{1}}(\mathbf{Y})$ and the edges associated with $C_{1, s}$ link $\mathbf{X}_{\mathbf{S}}(\mathbf{Y})$ to $\boldsymbol{\Upsilon}_{\mathbf{1}}(\mathbf{Y})$, we have that $g_{-} \operatorname{rank}\left(\begin{array}{c}A_{1, s} \\ C_{1, s}\end{array}\right)=\theta\left[\mathbf{X}_{\mathbf{S}}(\mathbf{Y}), \mathbf{X}_{\mathbf{1}}(\mathbf{Y}) \cup \boldsymbol{\Upsilon}_{\mathbf{1}}(\mathbf{Y})\right]$. According to Statement St3 of Lemma 6 in [3], g_rank $\left(\begin{array}{c}A_{1, s} \\ C_{1, s}\end{array}\right)=n_{s}$ and so the number of invariant zeros of $P(s)$ is 
equal to the number of invariant zeros of $P_{e}(s)$, where

$$
P_{e}(s)=\left(\begin{array}{cccccc}
A_{0,0}-s I_{n_{0}} & A_{0, s} & B_{0,0} & A_{0,1} & B_{0,1} & 0 \\
A_{s, 0} & A_{s, s}-s I_{n_{s}} & B_{s, 0} & A_{s, 1} & B_{s, 1} & 0 \\
C_{0,0} & C_{0, s} & D_{0,0} & C_{0,1} & D_{0,1} & 0 \\
0 & I_{n_{s}} & 0 & 0 & 0 & 0 \\
0 & 0 & 0 & A_{1,1}-s I_{n_{1}} & B_{1,1} & A_{1, s} \\
0 & 0 & 0 & C_{1,1} & D_{1,1} & C_{1, s}
\end{array}\right)
$$

Let us denote $P_{0}(s) \stackrel{\text { def }}{=}\left(\begin{array}{ccc}A_{0,0}-s I_{n_{0}} & A_{0, s} & B_{0,0} \\ A_{s, 0} & A_{s, s}-s I_{n_{s}} & B_{s, 0} \\ C_{0,0} & C_{0, s} & D_{0,0} \\ 0 & I_{n_{s}} & 0\end{array}\right)$ and $P_{1}(s) \stackrel{\operatorname{def}}{=}\left(\begin{array}{ccc}A_{1,1}-s I_{n_{1}} & B_{1,1} & A_{1, s} \\ C_{1,1} & D_{1,1} & C_{1, s}\end{array}\right)$.

Matrices $P_{0}(s)$ and $P_{1}(s)$ can be seen respectively as the pencil matrices of the square system denoted $\Sigma_{0}$ and the system denoted $\Sigma_{1}$, which has generically full column $n$-rank even after the deletion of an arbitrary row [3].

From [30], as $\rho\left[\mathbf{U}_{\mathbf{0}}(\mathbf{Y}), \mathbf{\Upsilon}_{\mathbf{0}}(\mathbf{Y}) \cup \mathbf{X}_{\mathbf{s}}(\mathbf{Y})\right]=\operatorname{card}\left(\mathbf{U}_{\mathbf{0}}(\mathbf{Y})\right), g \_n-r a n k\left(P_{0}(s)\right)$ is equal to the number of rows (or columns) of $P_{0}(s)$ since it is a square matrix. Furthermore, since $\rho\left[\mathbf{U}_{\mathbf{1}}(\mathbf{Y}) \cup\right.$ $\left.\mathbf{X}_{\mathbf{s}}(\mathbf{Y}), \Upsilon_{\mathbf{1}}(\mathbf{Y})\right]=\operatorname{card}\left(\mathbf{U}_{\mathbf{1}}(\mathbf{Y}) \cup \mathbf{X}_{\mathbf{s}}(\mathbf{Y})\right)$, we have also $g \_n$-rank $\left(P_{1}(s)\right)$ is equal to the number of columns of $P_{1}(s)$. Thus, counting the zeros with their multiplicities, the number of invariant zeros of $P_{e}(s)$ is equal to the sum of the number of invariant zeros of $P_{0}(s)$ and the number of invariant zeros of $P_{1}(s)$. On the one hand, applying Theorem 5.1 of [30], we have that the number of invariant zeros of $P_{0}(s)$ is equal to $n_{0}+n_{s}+q_{0}-\mu\left[\mathbf{U}_{\mathbf{0}}(\mathbf{Y}), \mathbf{S}^{\mathbf{o}}\left(\mathbf{U}_{\mathbf{0}}(\mathbf{Y}), \mathbf{Y}\right)\right]+\rho\left[\mathbf{U}_{\mathbf{0}}(\mathbf{Y}), \mathbf{S}^{\mathbf{o}}\left(\mathbf{U}_{\mathbf{0}}(\mathbf{Y}), \mathbf{Y}\right)\right]-n_{s}$. Note that the presence of the latter term $n_{s}$ is due to the fact that the output of system $\Sigma_{0}$ is $y_{s}(t)$ and not $x_{s}(t)$. On the other hand, from Theorem 5.2 of [30], the number of invariant zeros of $P_{1}(s)$ is equal to $n_{1}+n_{s}+q_{1}$ minus the maximal number of vertices of $\mathbf{X}_{\mathbf{1}}(\mathbf{Y}) \cup \mathbf{X}_{\mathbf{s}}(\mathbf{Y}) \cup \mathbf{U}_{\mathbf{1}}(\mathbf{Y})$ covered by a disjoint union of:

- a $\mathbf{X}_{\mathbf{s}}(\mathbf{Y}) \cup \mathbf{U}_{\mathbf{1}}(\mathbf{Y})-\Upsilon_{\mathbf{1}}(\mathbf{Y})$ linking of size $\rho\left[\mathbf{X}_{\mathbf{S}}(\mathbf{Y}) \cup \mathbf{U}_{\mathbf{1}}(\mathbf{Y}), \Upsilon_{\mathbf{1}}(\mathbf{Y})\right]$,

- a $\Upsilon_{1}(\mathbf{Y})$-topped path family and

- a cycle family covering only elements of $\mathrm{X}_{1}(\mathrm{Y})$.

Therefore, using notations of Definition 3, the number of invariant zeros of $P_{e}(s)$ and also of $P(s)$ is equal to $n_{0}+q_{0}+n_{1}+n_{s}+q_{1}-\beta_{0}(\mathbf{Y})-\beta_{1}(\mathbf{Y})=n+\bar{q}-\beta_{0}(\mathbf{Y})-\beta_{1}(\mathbf{Y})$. Thus, the generic dimension of the strongly observable subspace of $\Sigma_{\Lambda}$ in the extended state and input space is equal to $n+\bar{q}-g \_n_{i n v, z}=\beta_{1}(\mathbf{Y})+\mu\left[\mathbf{U}_{\mathbf{0}}(\mathbf{Y}), \mathbf{S}^{\mathbf{o}}\left(\mathbf{U}_{\mathbf{0}}(\mathbf{Y}), \mathbf{Y}\right)\right]-\rho\left[\mathbf{U}_{\mathbf{0}}(\mathbf{Y}), \mathbf{S}^{\mathbf{o}}\left(\mathbf{U}_{\mathbf{0}}(\mathbf{Y}), \mathbf{Y}\right)\right]=$ 
$\beta_{1}(\mathbf{Y})+\beta_{0}(\mathbf{Y})=\beta(\mathbf{Y})$

The previous lemma allows us to write that the generic dimension of the strongly observable subspace in the extended state and input space $\left(x^{T}(t), u^{T}(t)\right)^{T}$ is equal to $\beta(\mathbf{Y})$. The necessary and sufficient strong observability conditions can be deduced immediately from this Lemma. Indeed, if $\beta(\mathbf{Y})<n+q$ then $\Sigma_{\Lambda}$ is not generically input and state observable and it may be interesting to know which state component $x_{i}$ (resp. input component $u_{j}$ ) is generically strongly observable. At this aim, we compare $\beta\left(\mathbf{Y} \cup\left\{\mathbf{x}_{\mathbf{i}}\right\}\right)$ (resp. $\left.\beta\left(\mathbf{Y} \cup\left\{\mathbf{u}_{\mathbf{j}}\right\}\right)\right)$ to $\beta(\mathbf{Y})$. Indeed, this amounts to compare the generic dimension of the strongly observable subspace in the extended state and input space $\left(x^{T}(t), u^{T}(t)\right)^{T}$ of $\Sigma_{\Lambda}$ to the generic dimension of the strongly observable subspace in the extended state and input space $\left(x^{T}(t), u^{T}(t)\right)^{T}$ of the same system $\Sigma_{\Lambda}$ with an additional sensor which measures the component $x_{i}(t)$ (resp. $u_{j}(t)$ ). In fact, adding to the system a sensor, which measures the state component $x_{i}(t)$ (resp. input component $u_{j}(t)$ ) is equivalent to add in the digraph an output vertex $\mathbf{y}_{\mathbf{p}+\mathbf{1}}$ and an edge $\left(\mathbf{x}_{\mathbf{i}}, \mathbf{y}_{\mathbf{p}+\mathbf{1}}\right)$ (resp. $\left.\left(\mathbf{u}_{\mathbf{j}}, \mathbf{y}_{\mathbf{p}+\mathbf{1}}\right)\right)$. For the new system obtained by adding of $\mathbf{y}_{\mathbf{p}+\mathbf{1}}$, the computation of the generic dimension of the strongly observable subspace in the extended state and input space $\left(x^{T}, u^{T}\right)^{T}$ can be made by using function $\beta\left(\mathbf{Y} \cup\left\{\mathbf{y}_{\mathbf{p}+\mathbf{1}}\right\}\right)$. Nevertheless, this requires an effective redraw of the digraph to add effectively an output vertex $\mathbf{y}_{\mathbf{p}+\mathbf{1}}$ and an edge $\left(\mathbf{x}_{\mathbf{i}}, \mathbf{y}_{\mathbf{p}+\mathbf{1}}\right)\left(\operatorname{resp} .\left(\mathbf{u}_{\mathbf{j}}, \mathbf{y}_{\mathbf{p}+\mathbf{1}}\right)\right)$. For the sake of simplicity, we have chosen to work on an unique digraph. Thus, we do not add any vertex or edge in the digraph, but we consider vertex $\mathbf{x}_{\mathbf{i}}$ (resp. $\mathbf{u}_{\mathbf{j}}$ ) as an output. Thus, it is easy to deduce from Lemma 1 that $\beta(\mathbf{V})=\beta_{1}(\mathbf{V})+\mu\left[\mathbf{U}_{\mathbf{0}}(\mathbf{V}), \mathbf{S}^{\mathbf{o}}(\mathbf{V})\right]-\rho\left[\mathbf{U}_{\mathbf{0}}(\mathbf{V}), \mathbf{S}^{\mathbf{o}}(\mathbf{V})\right]+\operatorname{card}(\mathbf{V} \backslash \mathbf{Y})$, for $\mathbf{V}=\mathbf{Y} \cup\left\{\mathbf{x}_{\mathbf{i}}\right\}$ (resp. $\mathbf{V}=\mathbf{Y} \cup\left\{\mathbf{u}_{\mathbf{j}}\right\}$ ) represents the generic dimension of the strongly observable subspace in the extended state and input space $\left(x^{T}, u^{T}\right)^{T}$ for the new system obtained adding of a measurement on $x_{i}$ (resp. $u_{j}$ ). Hence, we have:

Proposition 1 Consider structured system $\Sigma_{\Lambda}$ represented by digraph $\mathcal{G}\left(\Sigma_{\Lambda}\right)$. Let $\mathcal{V}_{\text {obs }} \stackrel{\text { def }}{=}$ $\{\mathbf{v} \in \mathbf{X} \cup \mathbf{U}, \beta(\mathbf{Y} \cup\{\mathbf{v}\})=\beta(\mathbf{Y})\}$. State component $x_{i}(t)$ (respectively input component $u_{j}(t)$ ) is strongly observable iff $\mathbf{x}_{\mathbf{i}} \in \mathcal{V}_{\text {obs }}$ (resp. $\mathbf{u}_{\mathbf{j}} \in \mathcal{V}_{\text {obs }}$ ).

Proof: Obviously, a state component $x_{i}(t)$ is strongly observable iff an additional measure of this state component does not change the generic dimension of the strongly observable subspace. Using notations of Definition 3, this implies that state component $x_{i}(t)$ (resp. input component $u_{j}(t)$ ) is strongly observable iff $\beta(\mathbf{Y})=\beta\left(\mathbf{Y} \cup\left\{\mathbf{x}_{\mathbf{i}}\right\}\right)$ (resp. $\beta(\mathbf{Y})=\beta\left(\mathbf{Y} \cup\left\{\mathbf{u}_{\mathbf{j}}\right\}\right)$ ) and the proposition follows. 


\subsection{Computational aspects}

From a computational point of view, the previous main result of the paper is based on Proposition 1 which requires the computation of function $\beta$. The latter needs first a system decomposition as specified in Definition 3. This decomposition is done using $n+1$ computations of maximal linkings between two vertex subsets. The computation of a maximal size linking has a complexity order equal to $O\left(W^{2} \cdot M^{0.5}\right)$ using a transformation of the digraph into a flow graph [20], where $M=(n+q)(n+$ $q)+(n+q) p$ is the maximal number of edges and $W=n+p+q$ is the number of vertices in the digraph. The computation of function $\mu$ is done using the primal-dual algorithm [16]. The complexity of such algorithm is $O\left(W^{3} \cdot M\right)$. Next, the computation of $\beta_{1}$ is done after a transformation of the digraph by evaluating the maximal number of vertices covered by a maximal size linking. We can do this computation with a complexity order equal to $O\left(W^{3} \cdot M\right)$.

Finally, in the worst case, the overall complexity order to list all the strongly observable state and input components is equal to $O\left(W^{4} \cdot M\right)$. If we assume, without loss of generality, that $n>p$ and $n \geq q$, then the complexity order is $O\left(n^{6}\right)$. Even if they can still be optimized, the proposed algorithms do not have an exponential complexity. Hence, they are suited to large scale systems.

The second part of this paper, which is devoted to the distributed systems observability analysis, is based on the results provided above. It is important to notice that the latter have many other fields of application.For example, in the case of systems submitted to faults, it is interesting to see whether or not the state is (or remains) observable when the system is faulty. Denoting by $\mathbf{F}$ the fault vertices (as input vertices), the state is strongly observable w.r.t. to the fault iff $\beta(\mathbf{Y})=\rho[\mathbf{F}, \mathbf{Y} \cup \mathbf{X}]+n$. This result is quite simple to deduce from Proposition 1. Thus, the presented result on the partial observability is a point of departure of many studies concerning the generic observability or other structural properties of structured linear systems with unknown inputs.

\subsection{Application to distributed systems}

Consider now that the structured linear system $\Sigma_{\Lambda}$ is a decentralized system. It is then constituted of several subsystems $\Sigma_{R}^{i}, i=1, \ldots, N$. Each subsystem satisfies a model of the form (2). For both the decentralized interconnected and decentralized autonomous observation schemes, we want to know if the available measurements contain enough information to ensure the strong observability (or the reconstructibility) of a given set of state and input components. Naturally, as it is mentioned in Section 
2, even if all the measurements taken on the system are not necessarily available for each subsystem, we assume that locally, each observer can integrate the whole dynamics provided by the system's model. This implies that, for each subsystem, we do not consider, as in many other works, the state components of the other subsystems as unknown inputs. In fact, in our reasoning, the unknown inputs are only represented by vectors $u^{i}(t), i=1, \ldots, N$.

Hereafter, we begin by defining the digraph representing $\Sigma_{\Lambda}$ when the latter is constituted by $N$ subsystems of the form (2). This digraph is only an adaptation of the one defined in Section 3 to the distributed systems framework. In fact, a structured system $\Sigma_{\Lambda}$ constituted by subsystems $\Sigma_{R}^{i}$, $i=1, \ldots, N$ is represented by a digraph noted $\mathcal{G}\left(\Sigma_{\Lambda}\right)$ which is constituted by a vertex set $\mathcal{V}$ and an edge set $\mathcal{E}$. More precisely, $\mathcal{V}=\bigcup_{i=1}^{N}\left(\mathbf{X}^{\mathbf{i}} \cup \mathbf{U}^{\mathbf{i}} \cup \mathbf{Y}^{\mathbf{i}} \cup \widetilde{\mathbf{Y}}^{\mathbf{i}}\right)$, where $\mathbf{X}^{\mathbf{i}}=\left\{\mathbf{x}_{1}^{\mathbf{i}}, \ldots, \mathbf{x}_{\mathbf{n}_{\mathbf{i}}}^{\mathbf{i}}\right\}$ is the set of state vertices of subsystem $\Sigma_{R}^{i}, \mathbf{U}^{\mathbf{i}}=\left\{\mathbf{u}_{\mathbf{1}}^{\mathbf{i}}, \ldots, \mathbf{u}_{\mathbf{q}_{\mathbf{i}}}^{\mathbf{i}}\right\}$ is the set of input vertices of subsystem $\Sigma_{R}^{i}$ $\mathbf{Y}^{\mathbf{i}}=\left\{\mathbf{y}_{1}^{\mathbf{i}}, \ldots, \mathbf{y}_{\mathbf{p}_{\mathbf{i}}}^{\mathbf{i}}\right\}$ is the set of output vertices of subsystem $\Sigma_{R}^{i}$. We denote by $\tilde{\mathbf{Y}}^{\mathbf{i}} \subseteq \mathbf{Y} \backslash \mathbf{Y}^{\mathbf{i}}$ the set of output vertices associated with the measurements arriving through the network to subsystem $\Sigma_{R}^{i}$. The edge set is $\mathcal{E}=\bigcup_{i=1}^{N}\left(A_{i}\right.$-edges $\cup B_{i}$-edges $\cup C_{i}$-edges $\cup D_{i}$-edges $\bigcup_{j=1, j \neq i}^{N}\left(A_{i, j}\right.$-edges $\cup B_{i, j}$-edges $\cup C_{i, j}$-edges $\cup$ $D_{i, j}$-edges $\left.)\right)$, where $A_{i}$-edges $=\left\{\left(\mathbf{x}_{\ell}^{\mathbf{i}}, \mathbf{x}_{\mathbf{h}}^{\mathbf{i}}\right) \mid A_{i}(h, \ell) \neq 0\right\}, B_{i}$-edges $=\left\{\left(\mathbf{u}_{\ell}^{\mathbf{i}}, \mathbf{x}_{\mathbf{h}}^{\mathbf{i}}\right) \mid B_{i}(h, \ell) \neq 0\right\}$, $C_{i}$-edges $=\left\{\left(\mathbf{x}_{\ell}^{\mathbf{i}}, \mathbf{y}_{\mathbf{h}}^{\mathbf{i}}\right) \mid C_{i}(h, \ell) \neq 0\right\}, D_{i}$-edges $=\left\{\left(\mathbf{u}_{\ell}^{\mathbf{i}}, \mathbf{y}_{\mathbf{h}}^{\mathbf{i}}\right) \mid D_{i}(h, \ell) \neq 0\right\}$ and for $j \neq i$, $A_{i, j}$-edges $=\left\{\left(\mathbf{x}_{\ell}^{\mathbf{j}}, \mathbf{x}_{\mathbf{h}}^{\mathbf{i}}\right) \mid A_{i, j}(h, \ell) \neq 0\right\}, B_{i, j}$-edges $=\left\{\left(\mathbf{u}_{\ell}^{\mathbf{j}}, \mathbf{x}_{\mathbf{h}}^{\mathbf{i}}\right) \mid B_{i, j}(h, \ell) \neq 0\right\}, C_{i, j}$-edges $=$ $\left\{\left(\mathbf{x}_{\ell}^{\mathbf{j}}, \mathbf{y}_{\mathbf{h}}^{\mathbf{i}}\right) \mid C_{i, j}(h, \ell) \neq 0\right\}$, and $D_{i, j}$-edges $=\left\{\left(\mathbf{u}_{\ell}^{\mathbf{j}}, \mathbf{y}_{\mathbf{h}}^{\mathbf{i}}\right) \mid D_{i, j}(h, \ell) \neq 0\right\}$.

The definitions and notations given previously in Section 3 are obviously applicable to the distributed systems' case. Applying now conditions of Proposition 1 to a distributed system, we have:

Corollary 1 Consider structured system $\left(\Sigma_{\Lambda}\right)$ represented by digraph $\mathcal{G}\left(\Sigma_{\Lambda}\right)$ and constituted by subsystems $\Sigma_{R}^{i}, i=1, \ldots, N$. The components associated with a vertex subset $\Delta^{i} \subseteq \mathbf{X}^{\mathbf{i}} \cup \mathbf{U}^{\mathbf{i}}$ of subsystem $i$ is generically input and state observable in - a decentralized interconnected observation scheme iff

$$
\beta\left(\Delta^{\mathbf{i}} \cup \mathbf{Y}^{\mathbf{i}} \cup \tilde{\mathbf{Y}}^{\mathbf{i}}\right)=\beta\left(\mathbf{Y}^{\mathbf{i}} \cup \tilde{\mathbf{Y}}^{\mathbf{i}}\right)
$$

- a decentralized autonomous observation scheme iff

$$
\beta\left(\Delta^{\mathbf{i}} \cup \mathbf{Y}^{\mathbf{i}}\right)=\beta\left(\mathbf{Y}^{\mathbf{i}}\right)
$$

Moreover, subsystem $i$ is generically strongly observable in 
- a decentralized interconnected observation scheme iff

$$
\beta\left(\mathbf{X}^{\mathbf{i}} \cup \mathbf{Y}^{\mathbf{i}} \cup \tilde{\mathbf{Y}}^{\mathbf{i}}\right)=\beta\left(\mathbf{Y}^{\mathbf{i}} \cup \tilde{\mathbf{Y}}^{\mathbf{i}}\right)
$$

- a decentralized autonomous observation scheme iff

$$
\beta\left(\mathbf{X}^{\mathbf{i}} \cup \mathbf{Y}^{\mathbf{i}}\right)=\beta\left(\mathbf{Y}^{\mathbf{i}}\right)
$$

This corollary allows us to characterize the components which are strongly observable in each of the two considered observation schemes. The following example illustrates the simplicity and the applicability of the previous corollaries.

Example 3 The considered example is treated in [21] and is based on the system originally presented in [17]. It deals with a linearized model of a four-tank system. A scheme of this process is shown in [17] and is depicted in Figure 2 below.

The system has two inputs (pump throughput) which can be manipulated to control the water level

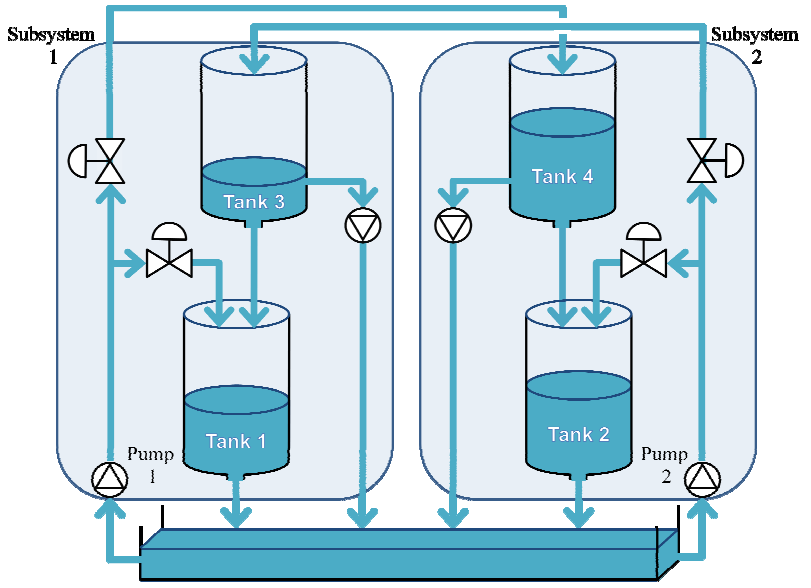

Figure 2. Four-tank system

in the tanks. The two pumps are used to transfer water from a basin into four overhead tanks. The two tanks at the upper level (Tank 3 and Tank 4) drain freely into the two tanks at the bottom level (Tank 1 and Tank 2) and the liquid levels in these bottom two tanks are measured by pressure sensors. The piping system is designed such that each pump affects the liquid levels of both measured tanks. A portion of the flow from one pump is directed into one of the lower level tanks and the rest is directed to the overhead tank that drains into the other lower level tank. The state variables are the four levels in the tanks and the speed of the flow that goes to the upper tank from each pump.

The system is divided into two symmetric subsystems. Each one is constituted by two tanks and their control input. For the first subsystem, we denote by $x_{1}^{1}(t)$ the liquid level in the bottom left tank, $x_{2}^{1}(t)$ 
the liquid level in the upper left tank, $x_{3}^{1}(t)$ the speed of the flow that goes to the upper left tank from the left pump, $u^{1}(t)$ the control signal related to the left pump. Similarly, for the second subsystem, we denote by $x_{1}^{2}(t)$ the liquid level in the bottom right tank, $x_{2}^{2}(t)$ the liquid level in the upper right tank, $x_{3}^{2}(t)$ the speed of the flow that goes to the upper right tank from the left pump and $u^{2}(t)$ the control signal related to the right pump. The measurements are the levels of two bottom tanks and their control input signals. The associated digraph is depicted in Figure 3.

We present only the computations on the first subsystem, knowing that they are exactly the same for

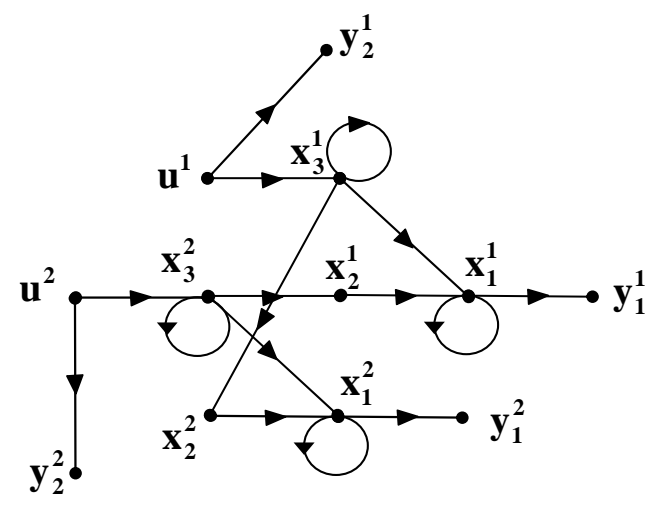

Figure 3. Digraph associated with four-tanks system

the second one. Some simple computations allow us to write that in a decentralized autonomous observation scheme $\mathbf{X}_{\mathbf{1}}^{\mathbf{1}}\left(\mathbf{Y}^{\mathbf{1}}\right)=\emptyset$ and $\mathbf{X}_{\mathbf{0}}^{\mathbf{1}}\left(\mathbf{Y}^{\mathbf{1}}\right)=\left\{\mathbf{x}_{\mathbf{1}}^{\mathbf{1}}, \mathbf{x}_{\mathbf{2}}^{\mathbf{1}}, \mathbf{x}_{\mathbf{3}}^{\mathbf{1}}\right\}$. As $V_{\text {ess }}\left(\mathbf{U}, \mathbf{Y}^{\mathbf{1}}\right)=\left\{\mathbf{u}_{\mathbf{1}}^{\mathbf{1}}, \mathbf{u}_{\mathbf{1}}^{\mathbf{2}}, \mathbf{x}_{\mathbf{3}}^{\mathbf{2}}, \mathbf{x}_{\mathbf{2}}^{\mathbf{1}}, \mathbf{x}_{\mathbf{1}}^{\mathbf{1}}\right\}$ does not contain $\mathbf{x}_{3}^{1}$, then, the state of subsystem 1 is not strongly observable. Let us determine the strongly observable state components of the first subsystem in a decentralized autonomous observation scheme. Yet, $\beta\left(\mathbf{Y}^{\mathbf{1}}\right)=\beta\left(\mathbf{Y}^{\mathbf{1}} \cup\left\{\mathbf{x}_{\mathbf{1}}^{\mathbf{1}}\right\}\right)=3$ and $\beta\left(\mathbf{Y}^{\mathbf{1}} \cup\left\{\mathbf{x}_{\mathbf{2}}^{\mathbf{1}}\right\}\right)=\beta\left(\mathbf{Y}^{\mathbf{1}} \cup\left\{\mathbf{x}_{\mathbf{3}}^{\mathbf{1}}\right\}\right)=5$. So, we can deduce that only state component $x_{1}^{1}(t)$ is strongly observable. Note that an additional measurement on $x_{2}^{1}(t)$ or $x_{3}^{1}(t)$ allows to recover the strong observability of the first subsystem in a decentralized autonomous observation scheme.

Furthermore, if one of the two measurements $y_{1}^{2}(t)$ or $y_{2}^{2}(t)$ is transmitted via the network to the first subsystem, the state of the first subsystem becomes strongly observable. Indeed, in this case, we have that $\mathbf{X}_{\mathbf{1}}^{\mathbf{1}}\left(\mathbf{Y}^{\mathbf{1}}\right)=\mathbf{X}^{\mathbf{1}}$ and $\theta\left[\mathbf{U} \cup \mathbf{X}, \mathbf{X}^{\mathbf{1}} \cup \mathbf{Y}^{\mathbf{1}} \cup \tilde{\mathbf{Y}^{\mathbf{1}}}\right]=6=3+\theta\left[\mathbf{U} \cup \mathbf{X}^{\mathbf{2}}, \mathbf{X} \cup \mathbf{Y}^{\mathbf{1}} \cup \tilde{\mathbf{Y}}^{\mathbf{1}}\right]=3+3$. This guarantees that all the state components of the first subsystem satisfy the conditions given in Proposition 1.

Now, let us consider briefly the case where it is not possible to implement the global model of the system in the local observers related to each subsystem. It amounts to consider for the $i^{t h}$ subsystem all the state components of the other subsystems as unknown inputs. To deal with such case, we consider 
a reduced graph $\mathcal{G}_{R}^{i}\left(\Sigma_{\Lambda}\right)$, where all the vertices $\mathbf{x}_{\mathbf{k}}^{\mathbf{j}}, j \neq i$ and $k=1, \ldots, n_{j}$ are transformed into input vertices and where all edges ending with these vertices are removed. The strong observability conditions of a state or an input component in a reduced observation scheme are then deduced from Corollary 1:

Corollary 2 Consider structured system $\left(\Sigma_{\Lambda}\right)$ represented by digraph $\mathcal{G}_{R}^{i}\left(\Sigma_{\Lambda}\right)$ defined above. The components associated with a vertex subset $\Delta^{\mathrm{i}} \subseteq \mathrm{X}^{\mathrm{i}} \cup \mathrm{U}^{\mathrm{i}}$ of subsystem $i$ are generically strongly observable in

- a reduced interconnected observation scheme iff

$$
\beta\left(\Delta^{\mathbf{i}} \cup \mathbf{Y}^{\mathbf{i}} \cup \tilde{\mathbf{Y}}^{\mathbf{i}}\right)=\beta\left(\mathbf{Y}^{\mathbf{i}} \cup \tilde{\mathbf{Y}}^{\mathbf{i}}\right)
$$

- a reduced autonomous observation scheme iff

$$
\beta\left(\Delta^{\mathbf{i}} \cup \mathbf{Y}^{\mathbf{i}}\right)=\beta\left(\mathbf{Y}^{\mathbf{i}}\right)
$$

Obviously, function $\beta$ is computed on digraph $\mathcal{G}_{R}^{i}\left(\Sigma_{\Lambda}\right)$ by considering as $\mathbf{U}_{\mathbf{e}} \stackrel{\text { def }}{=} \mathbf{U} \cup \mathbf{X} \backslash \mathbf{X}^{\mathbf{i}}$ input vertex subset.

\section{Concluding remarks}

In this paper, we first give, in graphical terms, necessary and sufficient conditions which ensure the strong observability of a state and/or an input component for a structured linear system. Next, we apply these conditions to study the generic observability of any given part of the states and the unknown inputs for network decentralized structured linear systems in both interconnected and autonomous schemes. Indeed, an important problem that has to be considered when dealing with control over network, is the validity of some properties like the observability. For network decentralized systems, an alternative to the centralized observation scheme, which can be quite complicated to consider when we deal with a large scale system, is to use a decentralized interconnected observation scheme or a completely decentralized autonomous observation scheme. The first one corresponds to the case where some subsystems are connected to the network and receive some information from the other subsystems. The second one is related to the case where all the subsystems have only their own measurement to reconstruct a part of the states and inputs.

All the proposed conditions need few information about the system and are easy to check by means 
of well-known combinatorial techniques and simply by hand for small systems. That makes our approach particularly suited for large-scale systems as it is free from numerical difficulties.

Another outlook to the proposed study is that we can easily deal with the optimisation of sensor location to achieve the strong observability of the system. It is also possible to easily determine which measurements are useful to transmit through the network to each subsystem in order to ensure the observability at less of some important variables. Finally, since the observability of a state or input component depends also on the subdivision of the system into subsystems, the present work can be used to design a tool in order to find the most pertinent decomposition from the observability point of view and further taking into account other structural properties.

\section{References}

[1] G. Basile and G. Marro. On observability of linear time-invariant systems with unknown inputs. Journal of Optimization Theory and Applications, 3(6):410-415, 1969.

[2] G. Basile and G. Marro. A new characterization of some structural properties of linear systems: unknown-input observability, invertibility and functional controllability. International Journal of Control, 17(5):931-943, 1973.

[3] T. Boukhobza, F. Hamelin, and S. Martinez-Martinez. State and input observability for structured linear Systems: a graph-theoretic approach. Automatica, 43(7):1204-1210, 2007.

[4] T. Boukhobza, F. Hamelin, and D. Sauter. Observability of structured linear systems in descriptor form: a graph-theoretic approach. Automatica, 42(4):629-635, 2006.

[5] D. Chu. Disturbance decoupled observer design for linear time-invariant systems: a matrix pencil approach. IEEE Transactions on Automatic Control, 45(8):1569-1575, 2000.

[6] D. Chu and V. Mehrmann. Disturbance decoupled observer design for descriptor systems. System \& Control Letters, 38(1):37-48, 1999.

[7] C. Commault, J. M. Dion, and V. Hovelaque. A geometric approach for structured systems: Application to disturbance decoupling. Automatica, 33(3):403-409, 1997.

[8] J. M. Dion, C. Commault, and J. W. van der Woude. Generic properties and control of linear structured systems: a survey. Automatica, 39(7):1125-1144, 2003.

[9] M.L.J. Hautus. Strong detectability and observers. Linear Algebra and its Applications, 50:353-360, 1983.

[10] M. Hou and P. C. Müller. Design of observers for linear systems with unknown inputs. IEEE Transactions on Automatic Control, 37(6):871-875, 1992.

[11] M. Hou and P. C. Müller. Design of decentralized linear state function observers. Automatica, 30(11):1801-1805, 1994.

[12] M. Hou and P. C. Müller. Causal observability of descriptor systems. IEEE Transactions on Automatic Control, 44(1):158-163, 1999. 
[13] M. Hou and P. C. Müller. Observer design for descriptor systems. IEEE Transactions on Automatic Control, 44(1):164-169, 1999.

[14] M. Hou and R. J. Patton. Input observability and input reconstruction. Automatica, 34(6):789-794, 1998.

[15] M. Hou, A. C. Pugh, and P. C. Müller. Disturbance decoupled functional observers. IEEE Transactions on Automatic Control, 44(2):382-386, 1999.

[16] V. Hovelaque, C. Commault, and J. M. Dion. Analysis of linear structured systems using a primal-dual algorithm. System \& Control Letters, 27:73-85., 1996.

[17] K. H. Johansson and J. L. R. Nunes. A multivariable laboratory process with an adjustable zero. In American Control Conference, pages 2045-2049, Philadelphia, USA, 1998.

[18] D. Koenig. Unknown input proportional multiple-integral observer design for linear descriptor systems: application to state and fault estimation. IEEE Transactions on Automatic Control, 50(2):212-217, 2005.

[19] P. Kudva, N. Viswanadham, and A. Ramakrishna. Observers for linear systems with unknown inputs. IEEE Transactions on Automatic Control, AC-25(1):113-115, 1980.

[20] S. Martinez-Martinez, T. Mader, T. Boukhobza, and F. Hamelin. LISA: A linear structured system analysis program. In Workshop de l'Institut Franco-Allemand Pour Les Applications de la Recherche (IAR/ACD), Nancy, France, 2006.

[21] M. Mercangöz and F. J. Doyle III. Distributed model predictive control of an experimental four-tank system. Journal of Process Control, 17(3):297-308, 2007.

[22] S. Micali and V. V. Vazirani. An $O\left(\left|V^{1 / 2} E\right|\right)$ algorithm for finding maximum matching in general graphs. In Proceedings of the $21^{\text {st }}$ Annual Symposium on the Foundations of Computer Science, pages 17-27, 1980.

[23] K. Murota. System Analysis by Graphs and Matroids. Springer-Verlag, New York, U.S.A., 1987.

[24] K. J. Reinschke. Multivariable Control. A Graph Theoretic Approach. Springer-Verlag, New York, U.S.A., 1988.

[25] M. Saif and Y. Guan. Decentralized state estimation in large-scale interconnected dynamical systems. Automatica, 28(1):215-219, 1992.

[26] C. W. Sanders, E. C. Tacker, and T. D. Lindon. A new class of decentralized filters for interconnected systems. IEEE Transactions on Automatic Control, AC-23(2):259-262, 1974.

[27] H. L. Trentelman, A. A. Stoorvogel, and M. Hautus. Control Theory for Linear Systems. Springer, London, U.K., 2001.

[28] H. Trinh and Q. Ha. Design of linear functional observers for linear systems with unknown inputs. International Journal of Systems Science, 31(6):741-749, 2000.

[29] C. C. Tsui. A new design approach to unknown input observers. IEEE Transactions on Automatic Control, 41(3):464-468, 1996.

[30] J. W. van der Woude. The generic number of invariant zeros of a structured linear system. SIAM Journal of Control and Optimization, 38(1):1-21, 2000.

[31] J. W. van der Woude, C. Commault, and J. M. Dion. Zero orders and dimensions of some invariant subspaces in linear structured systems. Mathematics of Control, Signals and Systems, 16(2-3):225-237, 2003. 